\title{
Strained Pt Schottky diodes on $n$-type Si and Ge
}

\author{
M. H. Liao \\ Department of Electrical Engineering and Graduate Institute of Electro-Optical Engineering, \\ National Taiwan University, Taipei, Taiwan, Republic of China
}

P.-S. Kuo and S.-R. Jan

Department of Electrical Engineering and Graduate Institute of Electronics Engineering, National Taiwan University, Taipei, Taiwan, Republic of China

\author{
S. T. Chang \\ Department of Electrical Engineering, National Chung Hsing University, Taichung, Taiwan, \\ Republic of China \\ C. W. Liu ${ }^{\text {a) }}$ \\ Department of Electrical Engineering, Graduate Institute of Electro-Optical Engineering, \\ and Graduate Institute of Electronics Engineering, National Taiwan University, Taipei, Taiwan, \\ Republic of China
}

(Received 7 November 2005; accepted 16 February 2006; published online 5 April 2006)

\begin{abstract}
The variation of electron barrier height and built-in voltage of Pt Schottky diodes on the mechanically strained $n$-type $\mathrm{Si}$ and $\mathrm{Ge}$ is investigated experimentally and theoretically. The mechanical strain is measured by Raman spectroscopy and analyzed by the finite element method. The built-in voltage and barrier height measured by capacitance-voltage and current-voltage methods, respectively, decrease with increasing external tensile strain. The reduction of the built-in voltage and barrier height originates mainly from the conduction band lowering with strain. The extracted value of conduction band lowering is consistent with the theoretical calculations using the "stress-free" boundary condition. ㅇ 2006 American Institute of Physics. [DOI: 10.1063/1.2191831]
\end{abstract}

Strained Si and Schottky barrier source/drain technologies have received considerable attention recently and can be used for high performance metal oxide semiconductor field effect transistors (MOSFETs) ${ }^{1,2}$ The substrate strain technology using the lattice misfit between $\mathrm{Si}$ and SiGe yields global biaxial strain and changes the conduction band and the valence band structure of Si. The carrier effective mass and the intervalley scattering are reduced, and thus the mobility is enhanced. Process and package induced strains can also produce sufficient strain for mobility enhancement at low cost. ${ }^{3,4}$ Schottky barriers improve the short channel effect due to the shallow junction. ${ }^{2}$ The previous study of the Schottky barrier height on the Pt/p-strained Si Schottky diode was reported. ${ }^{5}$ The external biaxial strain to reduce the Schottky barrier height and to increase complementary MOSFET drive current was also calculated by another group. ${ }^{6}$ In this Letter, the effect of externally mechanical strain on $n$-type Schottky barrier diodes on $\mathrm{Si}$ and $\mathrm{Ge}$ is presented experimentally and theoretically, and the shifts of the conduction band edge with mechanical strain are also studied.

The $n$-Si (100) and $n$-Ge (100) wafers used in this study were cleaned by dipping in dilute HF to remove the native oxide layer just before loading in the deposition chamber. Pt was deposited through a shadow mask with an area of $5 \times 10^{-3} \mathrm{~cm}^{2}$ by electron beam evaporation at a pressure of $2 \times 10^{-6}$ mbar to form the Schottky diodes. The Ohmic contact was made by thermal evaporation of $\mathrm{Al}$ on the back side of the wafer. The experimental setup to apply external mechanical strain to Schottky barrier diodes is similar to that

\footnotetext{
${ }^{\text {a) }}$ Author to whom correspondence should be addressed; electronic mail:
} chee@cc.ee.ntu.edu.tw described in Refs. 7 and 8. Two external strain conditions are applied in this work: (1) uniaxial tensile strain along the $\langle 110\rangle$ directions on (001) substrate and (2) biaxial tensile strain on (001) substrate. The level of strain is determined by the four screws on the sides of the washer. The strain of the $\mathrm{Si}$ and Ge under mechanical strain is simulated by finite element method (ANSYS) and measured by Raman spectroscopy. Raman spectra were excited by an Ar laser (wavelength of $514 \mathrm{~nm}$ ). The Ge-Ge peak in the Raman spectra (Fig. 1) shifts towards the negative axis under mechanical tensile strain. The Raman shifts of 0.91 and $1.32 \mathrm{~cm}^{-1}$ under uniaxial and biaxial tensile strains, respectively, were extracted from the curve fitting using a Lorentzian profile. The strain is obtained by ${ }^{9}$

$$
\Delta \omega=\frac{1}{2 \omega_{0}}\left[p \varepsilon_{z z}+q\left(\varepsilon_{x x}+\varepsilon_{y y}\right)\right] .
$$

For biaxial tensile strain,

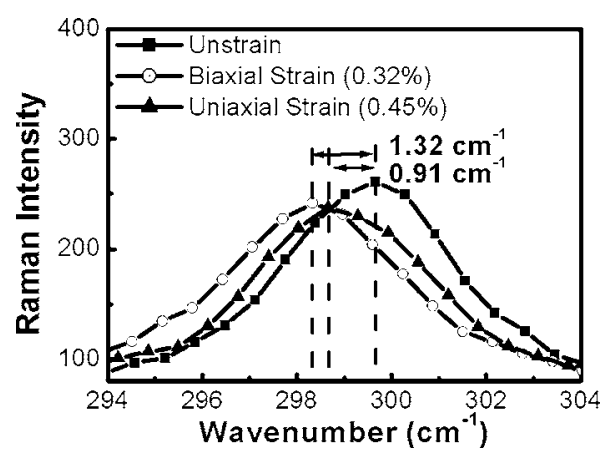

FIG. 1. Raman spectra of the mechanically strained Ge. The position of Ge-Ge peak of strained Ge indicates $0.32 \%$ for the biaxial tensile strain and $0.45 \%$ for the uniaxial tensile strain. 
TABLE I. The numerical value of parameters used in the calculation.

\begin{tabular}{lccccccccc}
\hline \hline & $\begin{array}{c}\mathrm{C}_{11} \\
(\mathrm{GPa})\end{array}$ & $\begin{array}{c}\mathrm{C}_{12} \\
(\mathrm{GPa})\end{array}$ & $\begin{array}{c}\mathrm{C}_{44} \\
(\mathrm{GPa})\end{array}$ & $\begin{array}{c}\mathrm{S}_{11} \\
(\mathrm{GPa})\end{array}$ & $\begin{array}{c}\mathrm{S}_{12} \\
(\mathrm{GPa})\end{array}$ & $\begin{array}{c}\mathrm{S}_{44} \\
(\mathrm{GPa})\end{array}$ & $\begin{array}{c}p \\
\left(10^{27} \mathrm{~s}^{-2}\right)\end{array}$ & $\begin{array}{c}\mathrm{q} \\
\left(10^{27} \mathrm{~s}^{-2}\right)\end{array}$ & $\begin{array}{c}\Xi_{d}+(1 / 3) \Xi_{u} \\
(\mathrm{eV})\end{array}$ \\
\hline $\mathrm{Si}_{(\Delta)}$ & 167 & 65 & 79 & 770 & -214 & 126 & -14.3 & -18.9 & 4.18 \\
$\mathrm{Ge}_{(L)}$ & 131 & 49 & 66 & 960 & -261 & 151 & -4.7 & -6.17 & -6.84 \\
\hline \hline
\end{tabular}

$$
\varepsilon_{z z}=-\left(2 C_{12}\right) /\left(C_{11}\right) \varepsilon_{x x}, \quad \varepsilon_{x x}=\varepsilon_{y y} .
$$

For uniaxial tensile strain,

$$
\begin{aligned}
& \varepsilon_{x x}=0.5 T\left(S_{11}+S_{12}\right)+0.25 T S_{44}, \\
& \varepsilon_{y y}=0.5 T\left(S_{11}+S_{12}\right)-0.25 T S_{44}, \\
& \varepsilon_{z z}=T S_{12},
\end{aligned}
$$

where $\omega_{0}$ is the longitudinal phonon frequency at the zone center of reciprocal space, $p$ and $q$ are phenomenological potentials to calculate the frequency shift as the function of external strain in Raman spectra, $\varepsilon_{x x}, \varepsilon_{y y}, \varepsilon_{z z}$ are the strain along the $\langle 110\rangle,\langle 1 \overline{1} 0\rangle,\langle 001\rangle$ directions on (100) wafer, $T$ is the stress, and $S_{11}, S_{12}, S_{44}, C_{11}$, and $C_{12}$ are elastic coefficients. The material parameters used in the calculation are given in Table I. ${ }^{9,10}$ In Table I, the $\Xi_{d}+(1 / 3) \Xi_{u}$ is the deformation potential constant to calculate the conduction band shift of Si and $\mathrm{Ge}$ as a function of external strain. Using the Raman shift of the Ge-Ge phonon peak in Fig. 1, the strain in Ge is estimated to be $0.32 \%$ for biaxial strain and $0.45 \%$ for uniaxial strain. Similarly, the strain in Si can also be estimated to be $0.13 \%$ for biaxial strain and $0.35 \%$ for uniaxial strain. ${ }^{7}$ The strain level obtained from Raman shift agrees well with the finite element simulation using ANSYS software.

Figure 2 shows the current-voltage $(I-V)$ characteristic of $\mathrm{Pt} / n-\mathrm{Si}$ and $\mathrm{Pt} / n-\mathrm{Ge}$ Schottky barrier diodes under biaxial tensile and uniaxial tensile mechanical strains. The barrier height and ideality factor $(n)$ can be calculated from the forward $I-V$ curves. The ideality factors of $n=1.07$ and $n=1.13$ for $\mathrm{Si}$ and Ge are observed, respectively, indicating that the current is mainly due to the thermionic emission. ${ }^{11}$ The barrier height changes of the $\mathrm{Pt} / n$-Ge sample are found to be -9 and $-27 \mathrm{meV}$ for uniaxial strain $(\sim 0.45 \%)$ and biaxial strain $(\sim 0.32 \%)$, respectively. For Pt $/ n$-Si diode, the changes are -8 and $-13 \mathrm{meV}$ for uniaxial strain $(\sim 0.35 \%)$ and biaxial strain $(\sim 0.13 \%)$, respectively. The Schottky barrier height decreases with increase of external mechanical

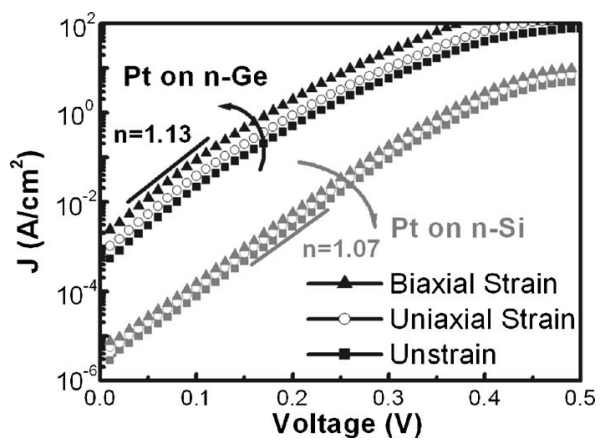

FIG. 2. Experimental forward $I-V$ characteristics of $\mathrm{Pt} / n-\mathrm{Si}$ and $\mathrm{Pt} / n-\mathrm{Ge}$ Schottky diodes with and without mechanical strain. Schottky diodes with and without mechanical strain.
Downloaded 13 Feb 2009 to 140.112113 .225 . Redistribution subject to AlP license or copyrig
FIG. 3. $C$ - $V$ characteristic of $\mathrm{Pt} / n$-Si and $\mathrm{Pt} / n$-Ge Schottky diodes with and

tensile strain. Neglecting the effect of surface dipole, the barrier height change of a Schottky junction under mechanical strain can be written as

$$
\Delta \phi_{b n}(\varepsilon)=\Delta \phi_{m}(\varepsilon)-\Delta \chi(\varepsilon),
$$

where $\phi_{b n}, \phi_{m}$, and $\chi$ represent barrier height, metal work function, and electron affinity of the semiconductor, respectively. The change of metal work function $\phi_{m}(\varepsilon)$ for single crystalline $\mathrm{Pt}$ is within $\sim 4 \mathrm{meV}$ under our mechanical strain conditions based on the derivation in Ref. 12.

$$
\frac{E_{F, \text { strain }}}{E_{F, \text { unstrain }}}=\left(\frac{V_{\text {unstrain }}}{V_{\text {strain }}}\right)^{2 / 3}
$$

where $V$ is the volume of metal and $E_{F}$ is the Fermi energy. Since the Pt in our sample is polycrystalline, the exact strain in the polygrains can be even smaller, and the change of the work function can be neglected. Therefore, the change of the Schottky barrier height under mechanical strain is mainly due to the change of the conduction band edge of the semiconductor.

Capacitance-voltage $(C-V)$ characteristics of $\mathrm{Pt} / n-\mathrm{Si}$ and $\mathrm{Pt} / n$-Ge samples under the biaxial tensile or uniaxial tensile strain are shown in Fig. 3. The doping concentrations obtained from the $\mathrm{Si}$ and $\mathrm{Ge}$ devices at reverse bias are $\sim 1 \times 10^{15}$ and $\sim 1 \times 10^{16} \mathrm{~cm}^{-3}$, respectively. The barrier height variation can be determined from ${ }^{11}$

$$
\Delta \phi_{b n}(\varepsilon)=\Delta V_{\mathrm{bi}}(\varepsilon)+\Delta\left(E_{C}-E_{F}\right)(\varepsilon),
$$

where $V_{\mathrm{bi}}(\varepsilon)$ is the built-in voltage measured from the voltage intercept of $C-V$, and $\left(E_{C}-E_{F}\right)(\varepsilon)$ is the depth of the Fermi level below the conduction band, which can be computed when the doping concentration is known,

$$
\Delta\left(E_{C}-E_{F}\right)(\varepsilon)=-K T \ln \left[\frac{n}{N_{c}(\varepsilon)}\right],
$$

where $N_{c}(\varepsilon)$ is the electron effective density of state as a function of strain in $\mathrm{Si}$ and $\mathrm{Ge}$, respectively. ${ }^{13}$ Based on $C-V$ measurement, the barrier height also decreases with external tensile strain.

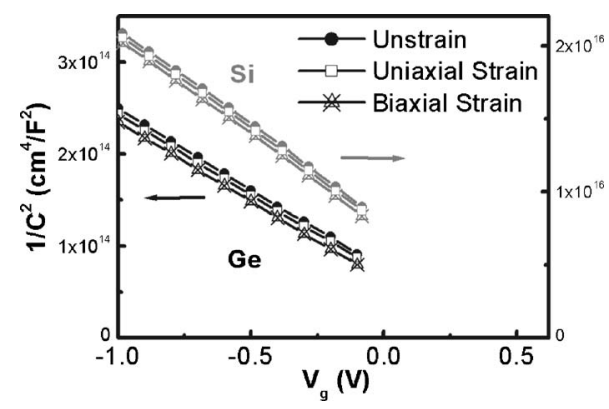

AlPlicense or copyright; see http://apl.aip.org/apl/copyright.jsp 


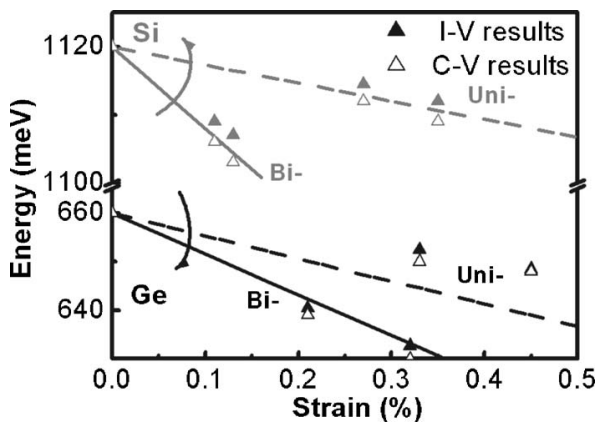

FIG. 4. Theoretical calculation and the experimental data of the shift of conduction band of $\mathrm{Si}$ and Ge under the external mechanical strain.

The theoretical calculation and experimental data of the conduction band shift in $\mathrm{Si}$ and $\mathrm{Ge}$ as a function of external strain along $\langle 110\rangle$ are shown in Fig. 4. Using the parameters in Refs. 10 and 14, and the formulas in Refs. 14 and 15, the shift of conduction band edge due to external tensile strain along $\langle 110\rangle$ is calculated under the stress-free condition. "Stress-free" means no stress along the in-plane direction perpendicular to the uniaxial strain axis $\langle 110\rangle$, i.e., $\langle 1 \overline{1} 0\rangle$ direction. The downshifts of $-23(-22) \mathrm{meV} / \%$ for the uniaxial strain and $-100(-84) \mathrm{meV} / \%$ for the biaxial strain are obtained for the $\mathrm{Si}(\mathrm{Ge})$ devices from $I-V$ measurement.

In summary, the reduction of the Schottky barrier height for the $n$-type semiconductor under external mechanical strain is observed. This reduction is shown to originate from the reduction of the conduction band edge. Using the stressfree boundary condition, a reasonable agreement between experimental data and theoretical calculation is obtained.
Raman measurements by Professor Chih-Ta Chia at the National Taiwan Normal University is highly appreciated. This work is supported by the National Science Council of ROC under Contract Nos. 94-2215-E-002-040 and 94-2215E-002-041, and the Asian Office of Aerospace Research and Development (AOARD), US Air Force.

${ }^{1}$ C. W. Liu, S. Maikap, and C.-Y. Yu, IEEE Circuits Devices Mag. 21, 21 (2005).

${ }^{2}$ M. Fritze, C. L. Chen, S. Calawa, D. Yost, B. Wheeler, P. Wyatt, C. L. Keast, J. Snyder, and J. Larson, IEEE Electron Device Lett. 25, 220 (2004).

${ }^{3}$ F. Yuan, C.-F. Huang, M.-H. Yu, and C. W. Liu, IEEE Trans. Electron Devices 53, 724 (2006).

${ }^{4}$ M. H. Liao, S. T. Chang, M. H. Lee, S. Maikap, and C. W. Liu, J. Appl. Phys. 98, 066104 (2005).

${ }^{5}$ S. Chattopadhyay, L. K. Bera, S. K. Ray, and C. K. Maiti, Appl. Phys. Lett. 71, 942 (1997).

${ }^{6}$ A. Yagishita, T.-J. King, and J. Bokor, Jpn. J. Appl. Phys., Part 1 43, 1713 (2004).

${ }^{7}$ M. H. Liao, M.-J. Chen, T. C. Chen, P.-L. Wang, and C. W. Liu, Appl. Phys. Lett. 86, 223502 (2005).

${ }^{8}$ M. H. Liao, C.-Y. Yu, C.-F. Huang, C.-H. Lin, C.-J. Lee, M.-H. Yu, S. T. Chang, C.-Y. Lee, T.-H. Guo, C.-C. Chang, and C. W. Liu, Tech. Dig. Int. Electron Devices Meet. 1023 (2006).

${ }^{9}$ Y. Hida, T. Tamagawa, H. Ueba, and C. Tatsuyama, J. Appl. Phys. 67, 7274 (1990).

${ }^{10}$ Properties of Crystalline Silicon, EMIS Data Reviews Series No. 20, edited by R. Hull (INSPEC, London, 1999).

${ }^{11}$ S. M. Sze, Physics of Semiconductor Devices (Wiley, New York, 1981).

${ }^{12}$ N. W. Ashcroft and N. D. Mermin, Solid State Physics (Harcourt Brace Jovanovitch, San Diego, 1975), pp. 32-38.

${ }^{13}$ M. V. Fischetti and S. E. Laux, J. Appl. Phys. 80, 2234 (1996)

${ }^{14}$ C. Ohler, C. Daniels, A. Forster, and H. Luth, Phys. Rev. B 58, 7864 (1998).

${ }^{15}$ J.-S. Lim, S. E. Thompson, and J. G. Fossum, IEEE Electron Device Lett. 25, 731 (2004). 